XXX. Kongress der Schweizerischen Gesellschaft für Dermatologie und Venereologie vom 9. und 10. Oktober 1948 in Basel

Dermatologica. 1949;98:302-303

\title{
Sur le psoriasis arthropathique
}

\begin{tabular}{|l|l|l} 
A. & Rétornaz \\
\hline
\end{tabular}

Clinique dermatologique universitaire Lausanne (Dir. Prof. Dr. H. Jaeger)

En analysant environ 400 observations de psoriasis traités à la Clinique dermatologique de Lausanne durant les quarante dernières années, nous avons pu rassembler 31 cas, soit le $8 \%$, où la dermatose est accompagnée de lesions articulaires.

Cette affection semble atteindre chez nous un peu plus fréquem-ment le sexe féminin (19 cas), alors que la plupart des etudes anté-rieures montrent une predominance nette du sexe masculin. Dans les $2 / 3$ de nos cas environ, le psoriasis apparaît pour la premiere fois entre 25 et 40 ans; il a en general un aspect et une distribution topo-graphique classiques; dans plusieurs cas cependant Céruption est atypique, soit dans sa localisation (psoriasis interverti, psor. des plis), soit dans son aspect (psor. suintant). Toutefois ces «atypies» ne sont pas constantes chez un même malade et ne s'observent pas à chaque poussée. Comme dans le psoriasis commun, la dermatose est ici essen-tiellement chronique et récidivante. Dans notre série de cas i'appa-rition de Гéruption est en general postérieure à celle des lesions articulaires; elle est le plus souvent parallèle dans son evolution aux poussées rhumatismales, mais elle peut en être aussi tout à fait indé-pendante. Parmi les cas que nous avons suivis personnellement, nous n'avons pas constate une resistance particulière à la thérapeutique externe habituelle, à $\Gamma$ exception des lesions siègeant sur les doigts et les orteils de malades atteints d'un rhumatisme déformant grave.

Les arthropathies s'installent le plus souvent insidieusement, frap-pent d'abord et le plus fréquemment seulement les petites articulations des extrémités. Le debut se fait en general par des douleurs articulaires (psoriasis douloureux!); puis surviennent des hvdrarthro-ses ou des infiltrats péri-articulaires à repetition qui peuvent conduire

Rétornaz, Sur le psoriasis arthropathique

303

dans les cas les plus sérieux au rhumatisme déformant (12 cas). L'ar-thrite a été mono-articulaire dans 3 cas, pluri-articulaire dans 20 cas et généralisée dans 8 cas.

L'étude radiologique montre d'une façon générale deux types de lesions articulaires: sur les petites articulations des extréniités ce sont des lesions d'arthrite ou de péri-arthrite chronique avec, dans les cas les plus graves, destruction partielle des surfaces articulaires et sub-luxations; sur les grosses articulations ce sont en general plutôt des images d'arthrose.

Un caractère tout à fait particulier de ce psoriasis arthopatique et qui permet de le séparer des autres rhumatismes articulaires chro-niques est $\Gamma$ absence dans Panamnèse de ces cas d'infections focales pré-existantes ainsi que celle de lesions du myocarde et de $\Gamma$ endocarde.

Les examens généraux du sang et des urines ont montré le plus souvent une acceleration de la vitesse de sedimentation des érythro-cytes et une augmentation du taux de la cholestérinémie. Conclusions 
L'analyse de 31 cas de psoriasis arthropathique montre que les lesions cutanées et articulaires de cette affection ont le plus souvent certains caractèies particuliers. La dermatose se distingue parfois par son evolution parallèle à celle des manifestations articulaires, parfois par une localisation atypique, plus rarement par une anomalie de Гélément éruptif ou par une resistance particulière à la thérapeu-tique dans certains territoires.

Quant aux lesions articulaires, si elles rapellent à premiere vue le rhumatisme articulaire chronique, elles sen distinguent cependant nettement par une localisation et une evolution spéciales et sur-tout par Tabsence d'infections focales et de lesions du myocarde ou de Tendocarde.

En tenant compte des arguments cliniques ci-dessus, il nous parait justifié de considérer le psoriasis arthropathique comme une entité clinique bien déíinie, mais dont $\Gamma$ étio-pathologie precise reste encore inconnue et très probablement dépendante de multiples facteurs. Cette etude sera publiée in extenso ultérieurement. 